\title{
Glatiramer Acetate-associated Refractory Immune Thrombocytopenic Purpura
}

\author{
$\underline{\text { Iftach Sagy }}^{1,2}$, Leah Shalev ${ }^{1,2}$, Itai Levi ${ }^{2,3}$, Elena Shleyfer ${ }^{1,2}$, Svetlana Valdman ${ }^{2,3}$, Leonid Barski ${ }^{1,2}$ \\ ${ }^{1}$ Department of Medicine F, Soroka University Medical Center, Beer-Sheva, Israel \\ ${ }^{2}$ Faculty of Health Sciences, Ben-Gurion University, Beer-Sheva, Israel \\ ${ }^{3}$ Department of Hematology, a University Medical Center, Beer-Sheva, Israel \\ ${ }^{4}$ Department of Neurology, Soroka University Medical Center, Beer-Sheva, Israel
}

Received: 27/01/2016

Accepted: $31 / 01 / 2016$

Published: $14 / 03 / 2016$

How to cite this article: Sagy I, Shalev L, Levi I, Shleyfer E, Valdman S, Barski L. Glatiramer acetate-associated refractory immune thrombocytopenic purpura. EJCRIM 2016;3:doi:10.12890/2016_000399

Conflicts of Interests: The authors declare that there are no competing interests.

This article is licensed under a Commons Attribution Non-Commercial 4.0 License

\section{ABSTRACT}

We present a case of glatiramer acetate-associated refractory immune thrombocytopenic purpura (ITP) in a female patient with multiple sclerosis. A search of MEDLINE/PubMed did not find any connection between glatiramer acetate and thrombocytopenia, specifically ITP. The autoimmune reaction was resistant to conservative ITP treatment, and was eventually managed only by splenectomy. To the best of our knowledge, this is the first report of glatiramer acetate-associated ITP. Physicians should be aware of this condition, and consider performing routine blood counts at the beginning of glatiramer acetate treatment.

\section{LEARNING POINTS}

- We present a unique case of glatiramer acetate-associated refractory immune thrombocytopenic purpura (ITP) in a female patient with multiple sclerosis, which was eventually managed only by splenectomy.

- Although glatiramer acetate is known for its immunomodulatory effect, a literature search did not reveal any reports of an association with ITP.

- Physicians should be aware of this condition, and consider performing routine blood counts at the beginning of and during glatiramer acetate treatment.

\section{KEYWORDS}

Glatiramer acetate; drug associated immune thrombocytopenic purpura; thrombocytopenia.

\section{INTRODUCTION}

Glatiramer acetate (Copaxone) is an immunomodulatory agent approved by the FDA for the treatment of relapsing-remitting multiple sclerosis (MS). It has been proposed that the mechanism of action of glatiramer acetate is by activation of T-lymphocyte suppressor cells and interference with pathological antigen presenting on the myelin sheath. The adverse effects of glatiramer acetate consist mostly of local site injection reactions along with transient systemic reactions. These reactions usually begin 30 sec to 30 min after agent injection, and consist of flushing, chest discomfort, palpitations, dyspnoea and anxiety. They are considered sporadic and self-limiting ${ }^{[1]}$. In a multi-centric double 
blind placebo controlled trial comparing glatiramer acetate to placebo, additional adverse events (apart from local and transient systemic reactions) occurred approximately equally in both groups. No routine ECG, laboratory blood counts or chemical tests are recommended for patient monitoring ${ }^{[2]}$.

Immune thrombocytopenia purpura (ITP) is an autoimmune disorder characterized by immunological destruction of otherwise normal platelets, leading to platelet counts of less than $100 \times 10^{\%} / \mathrm{L}$. ITP may occur in isolation (primary) or in association with other disorders (secondary) due to viral infections [such as hepatitis C (HCV), cytomegalovirus (CMV) or human immunodeficiency virus (HIV), systemic lupus erythematosus (SLE), lymph proliferative disorders and certain drugs ${ }^{[3]}$. We present a case of glatiramer acetate-associated refractory ITP in a female patient with multiple sclerosis.

\section{CASE REPORT}

A 40-year-old woman was admitted to hospital with a chief complaint of new onset rash. A day before her admission the patient had noticed an unusual transient bleeding while brushing her teeth. Her past medical history included multiple sclerosis diagnosed at the age of 27 and treated with interferon beta from the time of diagnosis. The patient was hospitalized a year before her current admission due to relapsing disease, which was treated with prednisone that was later tapered down. Two months before her latest admission the interferon beta was switched to glatiramer acetate by her neurologist because of postural tremor. This was the only medication she was prescribed during that period.

Upon arrival the patient was alert and without respiratory distress. Her temperature was $37.9^{\circ} \mathrm{C}$, heart rate was $95 / \mathrm{min}$, blood pressure was $147 / 90 \mathrm{mmHg}$ and oxygen saturation was $98 \%$ in room air. The physical examination was unremarkable except for petechial rash on her legs and shoulders and the previously diagnosed tremor associated with her primary disease.

Haemoglobin level was $12.1 \mathrm{~g} / \mathrm{dL}$, leukocyte count was $4,870 / \mathrm{mm}^{3}$ and platelet count was $1,000 / \mathrm{mm}^{3}$. In a routine outpatient blood count 2 months before her admission, the platelet count had been $263,000 / \mathrm{mm}^{3}$. A clotting test as well as serum electrolytes, AST, ALT, lipase, amylase and alkaline phosphatase were all within normal limits. LDH was 515 U/L. Antinuclear antibodies, rheumatoid factor, anticardiolipin, HCV, HIV, CMV IgM and EBV IgM were all negative. Bone marrow aspiration demonstrated normocellular marrow with multi-lobate forms of megakaryocytes, without evidence of tumour.

Glatiramer acetate was discontinued on admission. During her hospital stay, the patient had transient episodes of vaginal, oral, dermal and gastrointestinal bleeding. She remained haemodynamically stable, with a mild normocytic anaemia. All the bleeding episodes resolved conservatively under treatment with Hexakapron (tranexamic acid). The patient received prednisone $1 \mathrm{mg} / \mathrm{kg}$ from the time of her admission, together with a 4-day trial of dexamethasone $40 \mathrm{mg}$ daily. The patient was also treated with Rh0 (D) immune globulin $75 \mu \mathrm{g} / \mathrm{kg}$ and with a preceding trial of intravenous gamma globulins (IV IgG) $2 \mathrm{~g} / \mathrm{kg}$ without any change in platelet count.

Due to the therapeutic failure of all regimens mentioned above, the decision to perform a splenectomy was made because of the consequences of refractory symptomatic severe ITP. The patient was administered three courses of romiplostim for 3 weeks as a bridging therapy before the procedure, with lack of success in increasing platelet counts. After being vaccinated against pneumococcus, meningococcus and haemophilus influenza, the patient underwent splenectomy on the 37th day of hospitalization without complications. The platelet count started to increase immediately after the procedure. On discharge the platelet count was $328 / \mathrm{mm}^{3}$. The patient's neurologist has informed the manufacturer about the hospitalization and the suspected association with glatiramer acetate. Two years of haematological follow-up in the hospital outpatient clinic has revealed normal blood counts, without additional thrombocytopenia events or the need for further medical treatment for thrombocytopenia.

\section{DISCUSSION}

Herein we present the case of a patient who was admitted to hospital due to refractory symptomatic ITP, which was successfully managed only by splenectomy. Treatment for multiple sclerosis with glatiramer acetate had been initiated 2 months before her admission. This was the only treatment she was prescribed before the onset of the thrombocytopenia. Other causes of thrombocytopenia (such as viral infections, haematological and autoimmune conditions) were excluded. Thus, we can refer to this patient as having secondary drug-associated ITP. Distinguishing between drug-associated ITP and other thrombocytopenia aetiologies may be very challenging for the clinician. The incidence of this phenomenon is 10 cases per 1,000,000 persons per year and there are several possible mechanisms for this condition:

(a) hapten-dependent antibodies - in the case of penicillins or cephalosporins, the beta-lactam (hapten) binds to the autologous target site (cell membrane protein of the thrombocyte) to create an allergen;

(b) drug-induced platelet-reactive auto-antibodies - certain drugs generate antibodies against platelets without the presence of the drug (for instance, gold and procainamide); 
(c) drug-dependent antibodies - the forming antibodies destroy the platelets only when the drug, such as ranitidine, is present ${ }^{[4]}$. A systemic review of 247 case reports of drug-associated thrombocytopenia identified 98 different potential drugs. The most common drugs associated with thrombocytopenia were quinidine, gold and trimethoprim-sulfamethoxazole. Sporadic reports also mentioned penicillins, cephalosporins, NSAIDs, acetaminophen, digoxin, amiodarone and lithium. Of the 247 patients involved, $9 \%$ had a major bleed (defined as intracranial or retroperitoneal haemorrhage) and $0.8 \%$ died of bleeding ${ }^{[5]}$.

The clinical presentation of drug-associated ITP is mucocutaneous bleeding that begins 1-2 weeks, sometimes longer, after the patient has taken the sensitizing agent. Severe bleeding such as melena, haematuria and intracranial or retroperitoneal haemorrhage, is unusual and indicates severe thrombocytopenia of less than $20 / \mathrm{mm}^{3}$. The most important differential diagnosis is drug-associated thrombocytopenia (DIT). DIT is characterized by the same clinical features as ITP, starts after exposure to the sensitizing agent for at least a week and can be sometimes accompanied by neutropenia and haemolytic anaemia together with thrombocytopenia. After the suspected agent has been discontinued, the platelet count tends to return to normal within a week.

From the neurological aspect, it is worth mentioning that the association between MS and auto-immune diseases (e.g. diabetes, inflammatory bowel disease, rheumatoid arthritis, etc.) is well established. ITP was found to be 25 times more prevalent among MS patients compared with the general population. In addition, recently there have been reports that ITP can be linked to Interferon beta therapy in MS patients, along with more common haematological adverse effects such as a transient decrease in white blood cell counts. In this instance, the immunomodulatory effect of glatiramer acetate may be associated with the refractory thrombocytopenia in our patient. Unfortunately, since a routine laboratory kit to test for glatiramer acetate-associated antibodies is not available, we cannot establish the definitive diagnosis of immune thrombocytopenia purpura. The fact that cessation of the drug did not result in an instant increase in the platelet count, is another factor against a causal association between glatiramer acetate and thrombocytopenia. Nonetheless, in the absence of any other reasonable aetiology, the clinical suspicion for drug associated ITP still remains very high.

\section{REFERENCES}

1. Galetta SL, Markowitz C. US FDA-approved disease-modifying treatments for multiple sclerosis. CNS Drugs 2005;19:239-252.

2. Johnson K, Brooks B, Cohen J, et al. Copolymer 1 reduces relapse rate and improves disability in relapsing-remitting multiple sclerosis: results of a phase III multicenter, doubleblind, placebo-controlled trial. Neurology 1995;45:1268-1276.

3. Neunert C, Lim W, Crowther M, Cohen A, Solberg Jr L, Crowther MA. The American Society of Hematology 2011 evidence-based practice guideline for immune thrombocytopenia. Blood 2011;117:4190-4207.

4. van den Bemt PMLA, Meyboom RH, Egberts AC. Drug-induced immune thrombocytopenia. Drug Safety 2004;27:1243-1252.

5. George JN, Raskob GE, Shah SR, et al. Drug-induced thrombocytopenia: a systematic review of published case reports. Ann Intern Med 1998;129:886-890. 